\title{
Vom Kostenträger zum Fallmanager Krankenkassen müssen mitgestalten
}

\author{
Horst Braner \\ Gesundheitsökonom (FH) und Leiter Vertragsbereich Rheinland-Pfalz/Saarland, \\ Deutsche Angestellten Krankenkasse (DAK)
}

Von den Gesamtkrankheitskosten des Jahres 2002 in Höhe von 223,6 Milliarden Euro entfallen alleine $10 \%$ auf die Ausgaben für psychische Störungen (Abb. 1). Vom volkswirtschaftlichen Schaden, der durch den Ausfall von Arbeitskräften entsteht ganz zu schweigen (Tab. 1). Die Hauptursache hierfür liegt an der noch immer zu späten Identifizierung der psychisch Erkrankten und der hierdurch verpassten Chance, die Patienten rechtzeitig einer adäquaten Therapie zuzuführen und damit einer Chronifizierung vorzubeugen.

\section{Die Ausgangslage}

In einer Katamnesestudie der Deutschen Angestellten Krankenkasse (DAK) und der Allgemeinen Hospitalgesellschaft (AHG) aus dem Jahr 2004 wurde die Dauer zwischen Eintritt der Störung und einer krankheitsspezifischen Behandlung im Durchschnitt mit 7,29 Jahren ermittelt.

Wittchen et al. (2) zufolge können $41 \%$ aller Arbeitsunfähigkeitstage auf psychische Störungen zurückgeführt werden (Abb. 2). Bemerkenswert ist, dass in der allgemeinärztlichen Praxis ca. 35\% aller Störungen einen psychischen Ursprungs haben, die übrigen $65 \%$ entfallen auf körperliche Erkankungen (3). Laut Schätzungen dieser Studie werden aber lediglich $61 \%$ der psychischen Störungen als solche erkannt, $39 \%$ bleiben unerkannt. Von allen Personen mit einer psychischen Störung und einem entsprechenden Hilfesuchverhalten erhalten lediglich 36,4\% eine adäquate Behandlung (Abb. 3). Insofern darf es nicht verwundern, wenn die Patienten erst im Stadium der erfolgten Krankheitsmanifestierung bzw. Chronifizierung eine ihrer Erkrankung angemessene Zuwendung erfahren mit entsprechend langer Therapiedauer und ungünstiger Heilungsprognose.
Selbst in Zeiten der Entwicklung modernster medizinischer Technologien, Diagnostikverfahren oder Therapien gelingt es nach wie vor zu häufig nicht, arbeitsunfähig erkrankten Patienten/Versicherten den schnellen Wiedereinstieg in das Erwerbsleben zu ermöglichen. Die Folgen sind sowohl finanzielle als auch psychische Belastungen der Patienten. Aber auch den Kostenträgern entstehen vermeidbare Mehrausgaben durch langwierige Therapien, Zahlung von Lohnersatzleistungen oder durch Kosten für die Frühberentung.

\section{Das Ziel}

Das prioritäre Ziel aus Sicht der Kostenträger muss es daher sein,

- die Versorgungsabläufe zu analysieren,

- Defizite, Fehlallokationen und/oder Ineffizienzen im Schnittstellenmanagement zu identifizieren und

- durch Implementierung geeigneter Versorgungsstrukturen diese Schwachstellen zu minimieren bzw. im günstigsten Fall zu eliminieren.

Die Analyse muss dabei sowohl die Gründe für die Nichtbehandlung des genannten Personenkreises als auch die ggf. eingeleiteten Therapieschritte psychisch Erkrankter umfassen, die dann die Entwicklung von Lösungsstrategien ermöglichen.

Neben weiteren Gründen haben Wittchen et al. im Jahr 2001 für eine Nichtbehandlung das Nichtkennen bzw. Nichterkennen der

\section{Tab. 1 Volkswirtschaflticher Schaden durch Arbeitsunfähigkeit in} Deutschland, nach einer Studie der DAK und AHG 2004.

nach (4)

\section{jährlicher volkswirtschaftlicher Schaden in Zahlen}

durchschnittliche Krankheits- und Folgekosten je Patient gesamtwirtschaftlicher Schaden durch psychische Erkankungen

$40000 €$ entfallene Arbeitsjahre $4000000000 €$ entfallene Arbeitstage 157000 J. gesamtwirtschaftliches Einsparpotenzial 


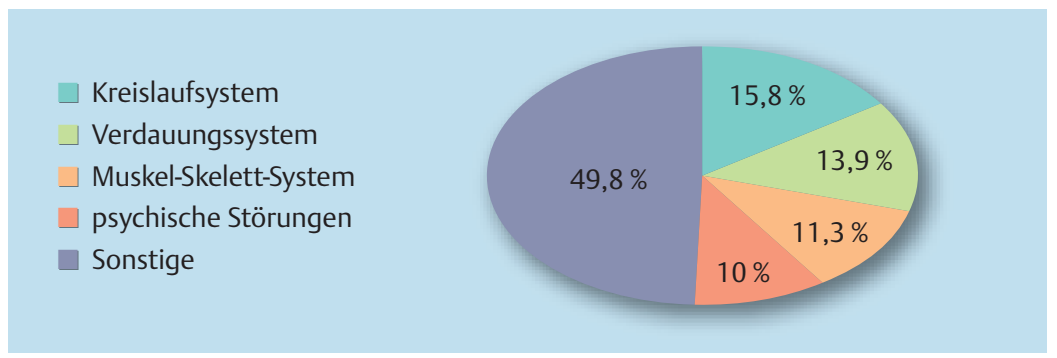

Abb. 1 Gesamtkrankheitskosten in 2002.

(Quelle: Statistisches Bundesamt)

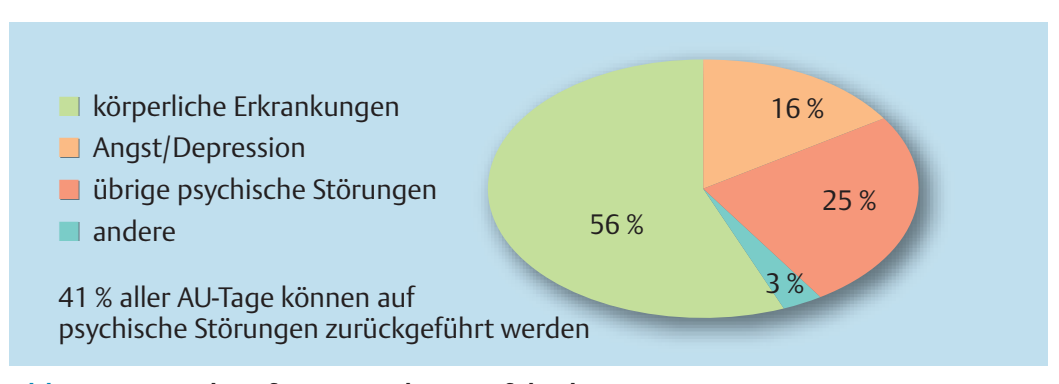

Abb. 2 Ursachen für eine Arbeitsunfähigkeit.

nach (3)

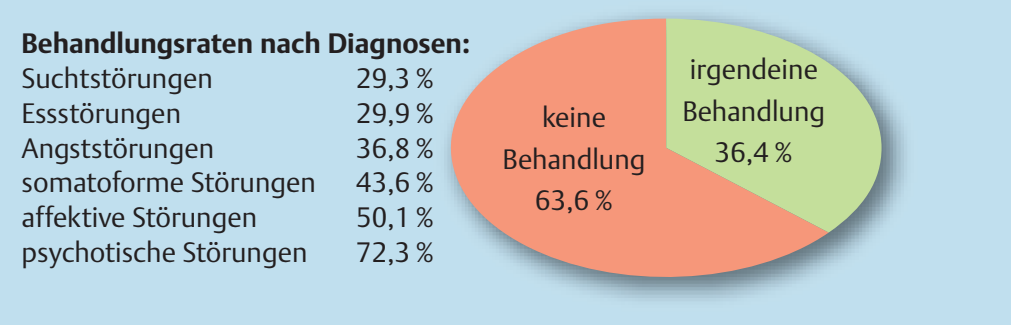

Abb. 3 Versorgungsraten und Art der Behandlung von Personen mit einer psychischen Störung und Hilfesuchverhalten.

nach (1)

Diagnose, die Ablehnung fachspezifischer Dienste, fehlende Nachhaltigkeit - trotz einer Überweisung wird die Therapie nicht aufgenommen - und die mangelnde Versorgungsdichte sowie lange Wartezeiten ausgemacht (3). Als Schlussfolgerung drängt sich demnach auf, dass es im allgemeinmedizinischen Bereich einer höheren Sensibilität für das Auftreten psychischer bzw. psychosomatischer Störungen bedarf. Zudem ist die

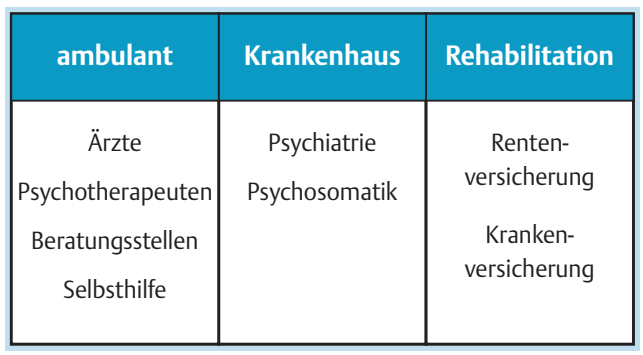

Abb. 4 Versorgungsstrukturen.

Kenntnis von über den eigenen Bereich hinausgehenden Hilfe- und Therapiemöglichkeiten sowie die Bereitschaft zur interdisziplinären und intersektoralen Kooperation von hoher Bedeutung.

\section{Die Intersektorale Versorgungstruktur}

Die derzeitige Versorgung psychisch Erkrankter wird durch den ambulanten Bereich, den stationären Bereich und die Rehabilitation der Renten- und Krankenversicherung abgedeckt, wobei eine intersektorale Abstimmung in der Regel nicht stattfindet (Abb. 4). Die heutigen, in sich geschlossenen Finanzierungsmechanismen mit ihren heterogenen Vergütungsformen der medizinischen Leistungen verleiten die Beteiligten dazu, die vorhandenen Versorgungsstrukturen entsprechend ihrer Finanzstärke bzw. Budgetzuordnung/-auslastung zu nutzen. Somit wird bewusst oder unbewusst eine Steuerung, die sich ausschließlich an dem optimalen Versorgungsgrad orientiert, verhindert. Eine effektive und effiziente Behandlung sowohl in therapeutischer als auch in finanzieller Hinsicht erfordert ein Aufbrechen dieser Sektoren und deren immanente Budgetverantwortlichkeit. Voraussetzungen sind unter anderem die Kooperationsbereitschaft der Akteure und der Aufbau einer transparenten und reibungslos funktionierenden Kommunikationsstruktur.

From the statutory sickness insurance body as the official cost bearer to medical co-management -

The sickness insurance bodies must participate in case manegement

In spite of present-day update medical knowledge and technology, diagnostics and therapies, we are often still at a loss to accomplish rapid rehabilitation of patients to enable them to resume gainful employment. Consequently, such patients are often in dire straits both financially and emotionally. This also involves cost problems due to protracted therapies and wage substitute payments, as well as manpower problems. This is mainly due to delays in the correct diagnosis of mental and psychologically conditioned states of disease which ultimately become chronic. This is possibly due to a lack of knowledge by general practitioners of the mental causes of somatic diseases. Additionally, the present German system of reimbursement of medical fees and of treatment cost by statutory health insurance bodies results in or promotes failures in adequate treatment, apart from the inappropriate separation of out- and inpatient care. The new option of integrated care may help to solve this problem.

Rehabilitation - mental disease - present drawbacks - integrated care - reinstatement 


\section{Ein Beispiel}

Einen Lösungsansatz bietet derzeit die Integrierte Versorgung, die eine sektorenübergreifende Therapie und Finanzierung ermöglicht. Alle Beteiligten stellen den Patienten in den Mittelpunkt und bringen ihr Fachwissen und ihre Erfahrung ein: Zum Vorteil des Patienten, der eigenen ärztlichen Weiterbildung und Qualifizierung und damit einer effizienten Versorgungsstruktur. In diesem Kontext sieht die Krankenkasse als Kostenträger die Lösung in der Entwicklung von interdisziplinären und sektorenübergreifenden Versorgungsnetzen, aber auch in der Optimierung bestehender Strukturen. Die Beteiligten in den Netzwerken verpflichten sich zu einer kooperativen, fachübergreifenden Zusammenarbeit, zur regelmäßigen Weiterbildung und zu einer qualitätsgesicherten Behandlung nach einheitlichen Leitlinien und abgestimmten und kontrollierten Behandlungspfaden.

Eine solche Struktur trägt nicht nur zu einer erfolgversprechenden Therapie im frühen Stadium der psychischen Störungen bei, sie bietet auch oder gerade Patienten mit chronischen Störungen kompetente umfassende Hilfe und unterstützt somit maßgeblich die Heilung und die frühzeitige Wiederherstellung der Arbeitsfähigkeit.
Literatur

1 Bassler M, Koletzko W, Sellhorn T. Fallmanagement psychischer Störungen. PsyCaseManagement der DAK - Unternehmen Leben, 2007

2 Kruse J, Schmitz N, Woller W et al. Warum übersieht der Hausarzt die psychischen Störungen seiner Patienten? Psychother Psychosom Med Psychol 2004; 54: 45-51

3 Wittchen HU, Jacobi F. Die Versorgungssituation psychischer Störungen in Deutschland - Eine klinisch-epidemiologische Abschätzung anhand des Bundesgesundheitssurveys 1998. Bundesgesundheitsblatt 2001; 44: 993-1000

4 Kooperatives Katamneseprojekt zwischen der DAK und den Psychosomatischen Fachkliniken Bad Dürkheim, Bad Pyrmont und Berus im AHG-Wissenschaftsrat. 2004. Ergebnisqualität und Gesundheitsökonomie verhaltensmedizinischer Psychosomatik in der Klinik

\section{Korrespondenz}

Horst Braner

Leiter des Vertragsgebietes

Rheinland-Pfalz/Saarland

Wilhelm-Theodor-Römheld-Str. 32

55130 Mainz

Horst.Braner@DAK.de 